\title{
Optical-resolution photoacoustic microscopy of ischemic stroke
}

Song Hu, Ernie Gonzales, Brian Soetikno, Enhao Gong, Ping Yan, et al.

Song Hu, Ernie Gonzales, Brian Soetikno, Enhao Gong, Ping Yan, Konstantin Maslov, Jin-Moo Lee, Lihong V. Wang, "Optical-resolution photoacoustic microscopy of ischemic stroke," Proc. SPIE 7899, Photons Plus Ultrasound: Imaging and Sensing 2011, 789906 (10 February 2011); doi: 10.1117/12.874366

SPIE. Event: SPIE BiOS, 2011, San Francisco, California, United States 


\title{
Optical-resolution photoacoustic microscopy of ischemic stroke
}

\author{
Song $\mathrm{Hu}^{1}$, Ernie Gonzales ${ }^{2}$, Brian Soetikno ${ }^{1}$, Enhao Gong ${ }^{1}$, Ping Yan ${ }^{2}, \mathrm{Konstantin} \mathrm{Maslov}^{1}$, \\ Jin-Moo Lee ${ }^{2, *}$, and Lihong V. Wang, \\ ${ }^{1}$ Department of Biomedical Engineering, Washington University in St. Louis, St. Louis, MO 63130, USA \\ ${ }^{2}$ Department of Neurology and the Hope Center for Neurological Disorders, Washington University School \\ of Medicine, St. Louis, MO 63110, USA \\ ${ }^{*}$ Correspondence should be addressed to JML (animal model) and LVW (photoacoustics).
}

\begin{abstract}
A major obstacle in understanding the mechanism of ischemic stroke is the lack of a tool to noninvasively or minimally invasively monitor cerebral hemodynamics longitudinally. Here, we applied optical-resolution photoacoustic microscopy (OR-PAM) to longitudinally study ischemic stroke induced brain injury in a mouse model with transient middle cerebral artery occlusion (MCAO). OR-PAM showed that, during MCAO, the average hemoglobin oxygen saturation $\left(\mathrm{sO}_{2}\right)$ values of feeder arteries and draining veins within the stroke core region dropped $\sim 10 \%$ and $\sim 34 \%$, respectively. After reperfusion, arterial $\mathrm{sO}_{2}$ recovered back to the baseline; however, the venous $\mathrm{sO}_{2}$ increased above the baseline value by $\sim 7 \%$. Thereafter, venous $\mathrm{sO}_{2}$ values were close to the arterial $\mathrm{sO}_{2}$ values, suggesting eventual brain tissue infarction.
\end{abstract}

Keywords: Optical-resolution photoacoustic microscopy, ischemic stroke, vascular anatomy, hemoglobin oxygen saturation, oxygen extraction fraction.

\section{INTRODUCTION}

Ischemic stroke refers to the death of brain tissue resulting from an inadequate supply of blood and oxygen, usually due to the thrombus of major cerebral arteries [1]. Stroke-induced brain injury may lead to body paralysis, communication difficulty, or vision impairment. Studying cerebrovascular responses to brain ischemia has been limited by a variety of imaging modalities that have either good tissue penetration but low resolution, or high resolution but requiring invasive preparations (open-skull windows). Here, we report a study of cerebrovascular hemodynamics in a mouse model of focal ischemia using a novel minimally invasive, high-resolution technique known as optical-resolution photoacoustic microscopy (OR-PAM).

Using OR-PAM [2, 3], we serially imaged cerebral blood vessels through the intact skull before, during, and after onehour transient middle cerebral artery occlusion (MCAO) in Swiss Webster mice up to 24 days following ischemia. Cerebral vessels were imaged using two different wavelengths to quantify the relative concentrations of oxy- and deoxyhemoglobin, from which the hemoglobin oxygen saturation $\left(\mathrm{sO}_{2}\right)$ and the oxygen extraction fraction (OEF) were calculated.

\subsection{System description}

\section{METHODS AND MATERIALS}

In OR-PAM (Fig. 1), the cerebral vasculature is irradiated through intact mouse skulls with a short-pulsed laser beam generated by a wavelength-tunable laser system, consisting of an Nd:YLF pump laser and a dye laser. Wideband ultrasonic waves are induced as a result of transient thermo-elastic expansion due to the laser excitation, and then detected by a high-frequency ultrasonic transducer. To maximize the sensitivity, the optical illumination and the ultrasonic detection in our system are configured confocally by an acoustic-optical beam combiner, where a right-angle prism and a rhomboid prism form a cube with a thin layer of silicone oil in between. This novel design offers high acoustic detection sensitivity. A microscopic objective lens is employed to achieve nearly diffraction-limited optical

Photons Plus Ultrasound: Imaging and Sensing 2011, edited by Alexander A. Oraevsky, Lihong V. Wang, Proc. of SPIE Vol. 7899, 789906 · @ 2011 SPIE · CCC code: 1605-7422/11/\$18 · doi: 10.1117/12.874366 
resolution $(\sim 2.6 \mu \mathrm{m}$ in clear media). The detailed system description and performance can be found in our paper included in the same proceeding [4].

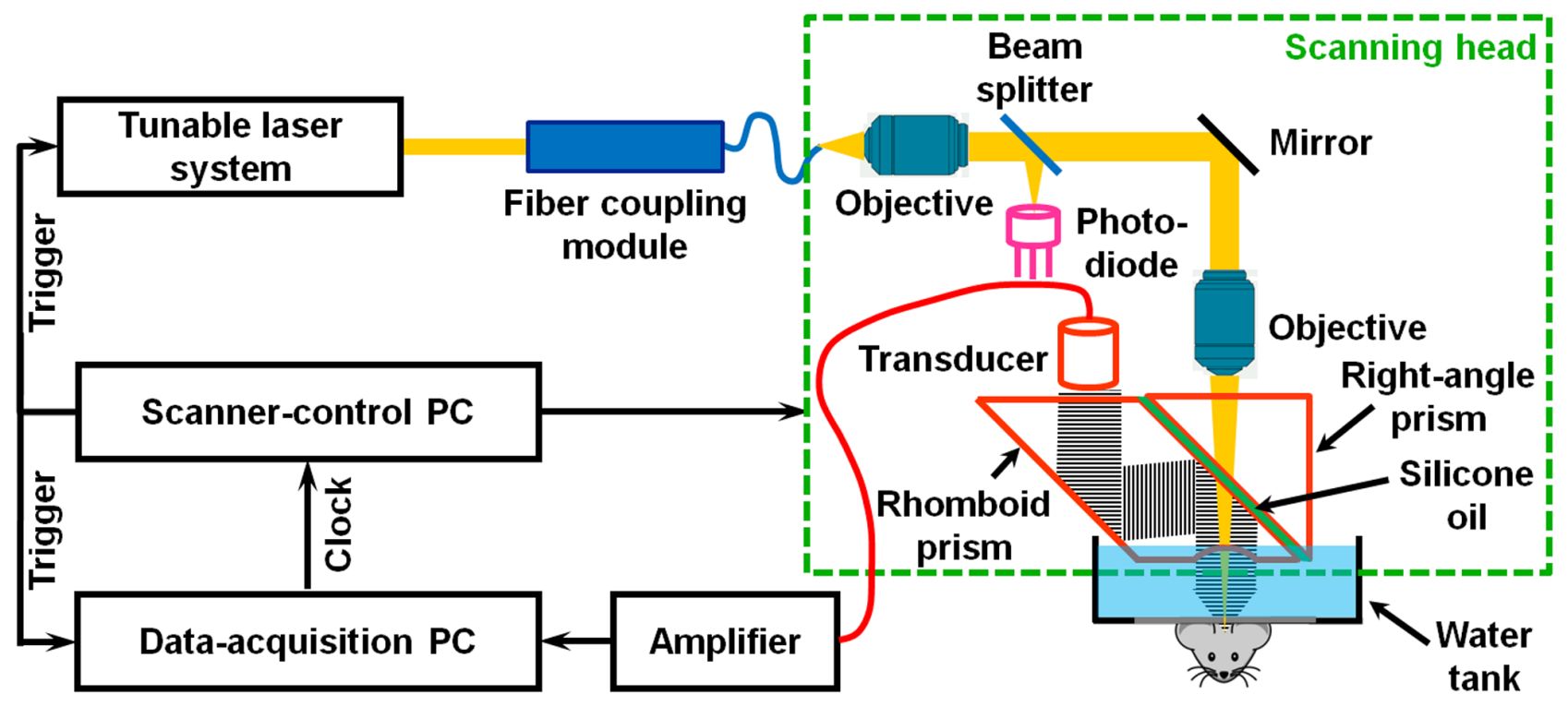

Figure 1. Schematic of the optical-resolution photoacoustic microscope system.

\subsection{Animal preparation}

A Swiss Webster mouse (Hsd:ND4, Harlan Co., $\sim 30 \mathrm{~g}$ ) was longitudinally monitored for up to 24 days post MCAO procedures. Before the first-day experiment, the mouse was anesthetized with intraperitoneal injection of an anesthesia cocktail [recipe: $1 \mathrm{ml}$ ketamine $(100 \mathrm{mg} / \mathrm{ml}), 0.1 \mathrm{ml}$ xylazine $(100 \mathrm{mg} / \mathrm{ml})$, and $8.9 \mathrm{ml}$ saline; dosage: $0.1 \mathrm{ml} / 10 \mathrm{~g}$ ]. Then the hair in the mouse head was shaved, and the residual hair was further depilated with Surgi Cream before cleaning with deionized water. Right before functional brain imaging at each monitoring point (i.e., before, during, after MCAO procedure, and 2 days, 7 days, and 24 days post MCAO), the experimental mouse was anesthetized with $3 \%$ isoflurane vaporized by the inhalation gas (flow rate: $1 \mathrm{l} / \mathrm{min}$ ), and then transferred to a custom-made stereotaxic imaging stage. The anesthesia was maintained with $1 \%$ isoflurane throughout the experiments. Medical-grade air was used as the inhalation gas to maintain the mouse at normal physiological status. The scalp of the mouse was surgically cut open along the sagittal line, and the exposed skull was cleaned with $0.9 \%$ sodium chloride irrigation solution right before imaging. Ultrasonic gel was used for ultrasound coupling and maintaining skull hydration. Throughout the experiment, the body temperature of the animal was maintained at $37^{\circ} \mathrm{C}$ by a temperature controlled heating pad. After each monitoring experiment, the scalp of the experimental mouse was sutured before applying antibacterial lotion. After 24day monitoring, the mouse was euthanatized for histology analysis of brain infarction.

\section{RESULTS}

Using dual-wavelength ( $563 \mathrm{~nm}$ and $570 \mathrm{~nm}$ ) OR-PAM, the vascular anatomy (Figs. 2A-2F) and $\mathrm{sO}_{2}$ (Figs. 2G-2L) were simultaneously monitored for 24 days following the MCAO-induced focal ischemia. The detailed method for $\mathrm{sO}_{2}$ computation can be found in our published papers [5,6]. It is clearly shown in the anatomical images that, during 60-min $\mathrm{MCAO}$, a majority of the microvessels in the stroke region disappeared, which indicated the MCAO-induced hypoperfusion of downstream microvasculature (Fig. 2B). After releasing the suture blocking the MCA blood flow, some of the microvessels showed up again, indicating the reperfusion of MCA (Fig. 2C). Starting from two days following ischemia, we can clearly see the dilation of draining veins, which was likely due to the accumulation of $\mathrm{CO}_{2}$ in the blood stream (Figs.2D-2F). More interestingly, from the longitudinal $\mathrm{sO}_{2}$ monitoring, we can clearly see that arteries could be easily distinguished from veins based on the baseline image shown in Fig. $2 \mathrm{G}$ (average arterial $\mathrm{sO}_{2}$ : 0.86; average 
venous $\left.\mathrm{sO}_{2}: 0.73\right)$. During $\mathrm{MCAO}$, the average $\mathrm{sO}_{2}$ values of arteries and veins within the stroke core region dropped $\sim 10 \%$ and $\sim 34 \%$, respectively (Fig. $2 \mathrm{H}$ ). After reperfusion, arterial $\mathrm{sO}_{2}$ recovered back to baseline; however, the venous $\mathrm{sO}_{2}$ increased above the baseline value by $\sim 7 \%$ (Fig. 2I). Thereafter, venous $\mathrm{sO}_{2}$ values were close to the arterial $\mathrm{sO}_{2}$ values (Figs.2J-2L).
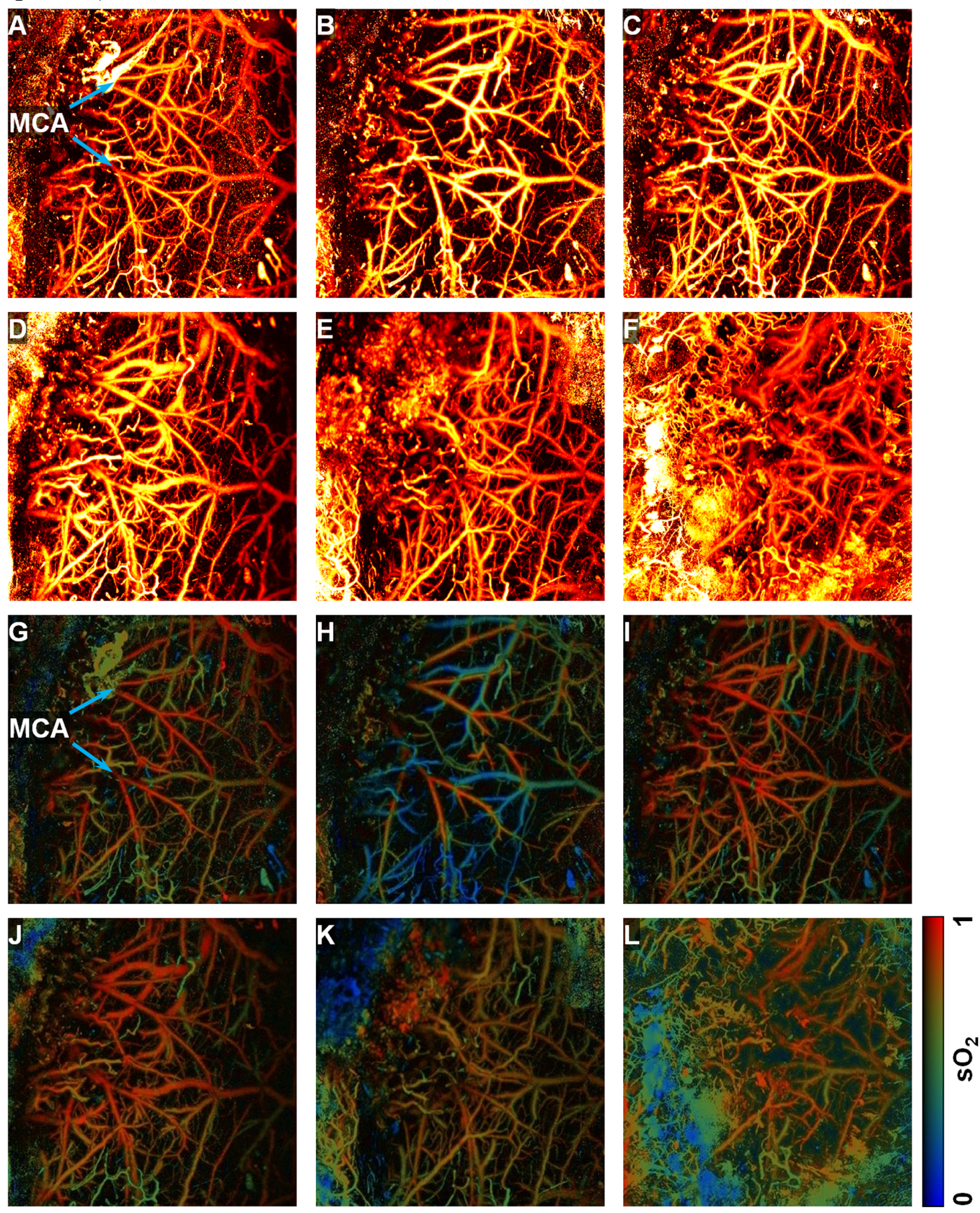

$500 \mu \mathrm{m}$

Figure 2. Longitudinal transcranial OR-PAM monitoring of (A-F) cerebral vascular anatomy and (G-L) hemoglobin oxygen saturation $\left(\mathrm{sO}_{2}\right)$ during stroke-induced ischemic brain injury. 
Further quantification of $\mathrm{OEF}$ based on the average $\mathrm{sO}_{2}$ values of the MCA and draining veins showed that, the OEF of the stroke region increased dramatically from 0.15 (baseline) to 0.48 during the MCAO to compensate for the decreased cerebral blood flow. After MCA reperfusion, the OEF progressively dropped and maintained at near-zero levels throughout the 24-day monitoring, which indicated the stroke-induced death of brain tissues.

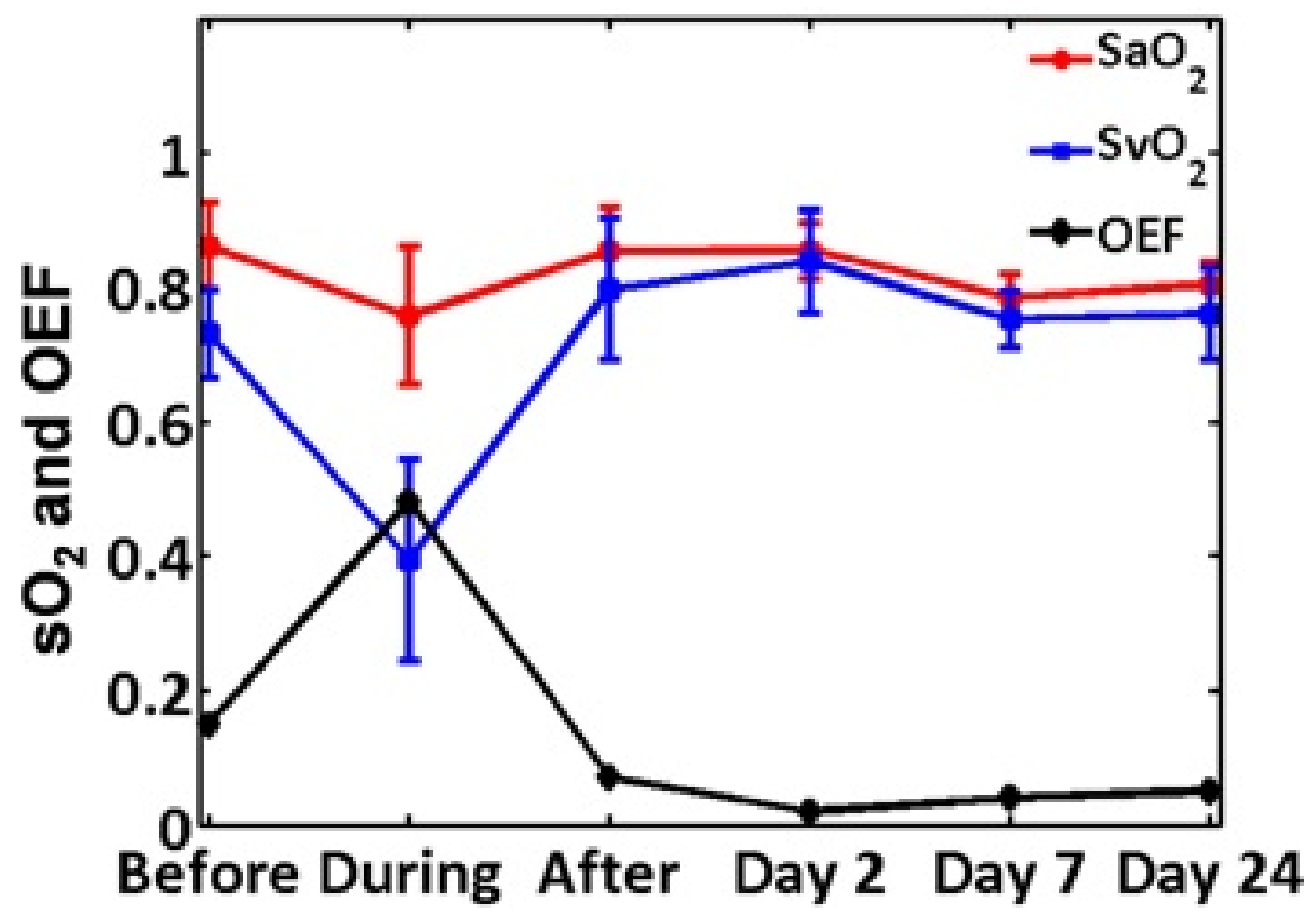

Figure 3. Longitudinal transcranial OR-PAM monitoring of oxygen extraction fraction (OEF) changes due to stroke-induced ischemic brain injury. $\mathrm{SaO}_{2}$ and $\mathrm{SvO}_{2}$ are the average $\mathrm{sO}_{2}$ in $\mathrm{MCA}$ and the draining veins, respectively.

\section{CONCLUSION}

We demonstrate, for the first time, longitudinal transcranial OR-PAM monitoring of morphological (i.e., vessel diameter) and functional (i.e., $\mathrm{sO}_{2}$ and $\mathrm{OEF}$ ) hemodynamic alterations during transient focal ischemia in mice. Consistent with previous PET studies in human stroke [7], we found OEF increased during acute ischemia, but progressively decreased after reperfusion, suggesting eventual brain tissue infarction. OR-PAM can be a valuable tool to longitudinally study cerebrovascular hemodynamics in intact mouse models of ischemic stroke. 


\section{ACKNOWLEDGMENTS}

This work was sponsored by National Institutes of Health Grants R01 EB000712, R01 EB008085, R01 CA134539, R01 NS48283, P01 NS32636, U54 CA136398, and 5P60 DK02057933. Prof. Lihong V. Wang has a financial interest in Microphotoacoustics, Inc. and Endra, Inc., which, however, did not support this work.

\section{REFERENCES}

[1] J. M. Lee, G. J. Zipfel and D. W. Choi, "The changing landscape of ischaemic brain injury mechanisms," Nature 399, A7-14 (1999).

[2] S. Hu, K. Maslov, V. Tsytsarev and L. V. Wang, "Functional transcranial brain imaging by optical-resolution photoacoustic microscopy," J. Biomed. Opt. 14(4), 040503 (2009).

[3] K. Maslov, H. F. Zhang, S. Hu and L. V. Wang, "Optical-resolution photoacoustic microscopy for in vivo imaging of single capillaries," Opt. Lett. 33(9), 929-931 (2008).

[4] K. Maslov, S. Hu and L. V. Wang, "Second-generation optical-resolution photoacoustic microscopy," SPIE Proceeding, (in press).

[5] S. Hu and L. V. Wang, "Photoacoustic imaging and characterization of the microvasculature," Journal of Biomedical Optics 15(1), 011101 (2010).

[6] H. F. Zhang, K. Maslov, M. Sivaramakrishnan, G. Stoica, and L. V. Wang, "Imaging of hemoglobin oxygen saturation variations in single vessels in vivo using photoacoustic microscopy," Applied Physics Letters 90(5), 053901 (2007).

[7] W.-D. Heiss, "Ischemic Penumbra: Evidence From Functional Imaging in Man," Journal of Cerebral Blood Flow \& Metabolism 20, 1276-1293 (2000). 